\title{
Wetting of doubly periodic rough surfaces in Wenzel's regime
}

\author{
Stanimir Iliev ${ }^{1, *}$, Nina Pesheva ${ }^{1}$, Pavel Iliev² \\ ${ }^{1}$ Institute of Mechanics, Bulgarian Academy of Sciences, 1113 Sofia, Bulgaria \\ ${ }^{2}$ ETH Zurich, Computational Physics for Engineering Materials, CH-8093 Zurich, Switzerland
}

\begin{abstract}
In this work we present preliminary results from our numerical study of the shapes of a liquid meniscus in contact with doubly sinusoidal rough surfaces in Wenzel's wetting regime. Using the full capillary model we obtain the advancing and the receding equilibrium meniscus shapes for a broad interval of surface roughness factors. The contact angle hysteresis is obtained when the three-phase contact line is located on one row (block case) or several rows (kink case) of physical defects. We find that depending on the mutual disposition of the contact line and the lattice of periodic defects, different stick-slip behaviors of the contact line depinning mechanism appear, leading to different values of the contact angle hysteresis.
\end{abstract}

\section{Introduction}

For various industrial applications one needs to devise solid surfaces with prescribed wettability properties. The surface wettability can be influenced through the formation of regularly spaced surface defects. These defects can be either physical (i.e., rough surfaces), and/or chemical defects (i.e., heterogeneous surfaces). That is why the study of the wetting properties of structured surfaces is very important. The presence of surface defects is known to lead to a set of metastable equilibrium states of the liquid, wetting the solid surface. Due to the presence of these defects, the liquid-vapor interface may move with the solid surface while the three-phase contact line (CL) is pinned at the surface defects and stays immobile relative to the solid surface. This phenomenon can lead to the increase or decrease of the apparent contact angle (CA). For the wetting properties of such solid surfaces is characteristic the so-called stick-slip behavior of the CL and the existence of maximal and minimal apparent contact angles, called the advancing and the receding CAs $\theta_{a d v}$ and $\theta_{r e c}$ (ACA and RCA). These CAs are attained just before the CL unpins, thus resulting in a displacement of the whole liquid meniscus. The difference of the advancing and the receding CAs is called $\mathrm{CA}$ hysteresis $(\mathrm{CAH})$, which is an important characteristic of the surface wettability. In the case of physical defects, i.e., rough surfaces, the ACA and RCA also depend on whether or not air is trapped between the solid surface and the liquid. The first case, when there is a trapped air, is known as Cassie's regime and the second case,

*Corresponding author: stani@imbm.bas.bg 
when there is no trapped air, as Wenzel's wetting regime. There are two possible types of CLs in respect to the rows of defects, these are: the straight line and the stepped contact line (terms, introduced in Ref. [1]), and the mechanisms of transition to the new equilibrium meniscus state are termed the block- and the kink-depining of the CL, respectively $[2,3]$. Here, we focus our study on the effect of the CL-depinning mechanism on the CAH in the case of smooth physical defects in Wenzel's wetting regime.

\section{Problem formulation}

\subsection{System}

We are concerned here with the study of the following system: a vertical solid plate, immersed in a tank of liquid (see Figure 1).

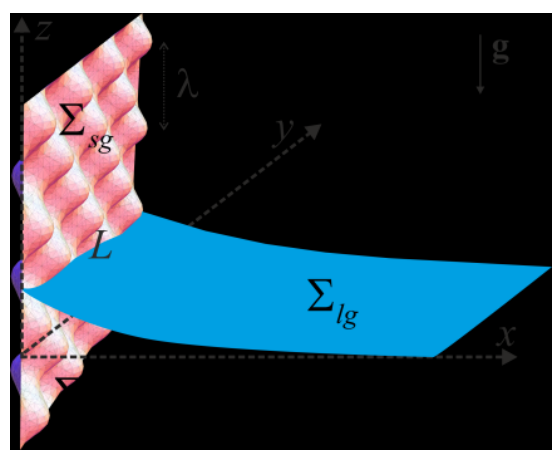

Fig. 1. Schematic image of the system under study: a 3D liquid meniscus, attached to a vertical rough solid plate.

More precisely, our goal is the determination of the set of metastable equilibrium shapes of the liquid meniscus in contact with the vertical plate, whose surface is rough and chemically homogeneous. The liquid in the tank has density $\rho$, volume $V$ and free surface $\Sigma_{l g}$. The rough solid plate, whose surface is denoted by $\Sigma_{s}$, has contact area $\Sigma_{s l}$ with the liquid ( $\Sigma_{s l} \subset \Sigma_{s}$ ), and we assume that, the wetting of the plate by the liquid, occurs in the Wenzel's wetting regime. The rough surface of the solid plate in Cartesian coordinates is described by the function $f(y, z)$, i.e., $\Sigma_{s} \equiv(f(y, z), y, z)$, where the y-axis is horizontal and the z-axis is directed upward, as shown in Fig. 1. The $(z=0)$-plane is chosen to coincide with the liquid level far from the moving plate. The function $f(y, z)$, describing the rough surface, is doubly periodic function of its coordinates with the same period $\lambda$ along the $y$ - and the z-axes. Here we concentrate on two types of such surfaces, for which one has

$$
f(y, z)=A \sin (2 \pi y / \lambda) \sin \left(2 \pi z / \lambda+z_{0}\right) .
$$

The values of the ratio $A / \lambda$, considered here, are of the order $0-0.2$ and the parameter $z_{0}$ (the vertical displacement of the plate, relative to the pool of liquid) varies in the interval $[0, \lambda)$. 
For obtaining the metastable equilibrium states of the meniscus under the action of the gravitational and capillary forces we adopt the integral approach. In this work we consider the case when the meniscus forms with the solid surface a local equilibrium CA $\theta_{e q}=90^{\circ}$. Thus we need to find the local minima of the system's free energy $U$, defined as

$$
U=\int_{\Sigma_{l g}} d \Sigma_{l g}+l_{c}^{2} \int_{V} z d V,
$$

where $l_{c}=\sqrt{\gamma / \rho g}$ is the capillary length, $\gamma$ is the liquid-gas surface tension, $g$ is the gravitational acceleration.

\subsection{Numerical method}

We obtain the metastable equilibrium meniscus states, using the minimization algorithm for the free energy $U$ (Eq. 2), described in detail in Refs [4-6]. For finding a numerical solution, the liquid free surface $\Sigma_{l g}$ is approximated by a set of triangles with $N_{i} \times N_{j}$ nodes with radius vectors $\mathbf{r}_{i j}$. The nodes $\mathbf{r}_{i j}$ of the triangles, approximating the meniscus shape, are shifted vertically and the nodes $\mathbf{r}_{i 1}$, approximating the CL, are shifted along the solid surface, each in direction minimizing the system energy $U$. We check the precision of our numerical method by comparing the results, obtained for different values of the displacements and for different number of the nodes $N_{i}, N_{j}$. On a length $\lambda$ we use in the present study between $30-90$ points. The numerical method is similar to the one, used in the public domain software "Evolver" [7].

\subsection{Apparent CA and CAH}

For the system, shown schematically in Fig. 1, the apparent CA $\theta_{a p}$ can be determined [3] through the observed height $h(L)$ of the CL $L$ :

$$
\theta_{a p}=\arcsin \left(1-\langle h(L)\rangle^{2} / 2 l_{c}^{2}\right) .
$$

For the system under consideration, $\theta_{r e c}$ and $\theta_{a d v}$ are attained, as follows from Eq. 3, for equilibrium meniscus states with the highest and the lowest capillary rise, respectively. Therefore, in order to obtain the whole set of equilibrium meniscus states and the CAH we proceed in the following way. First, we obtain numerically an equilibrium meniscus shape with an initial equilibrium CL, which for the study of the CL block depinning, is situated on one horizontal row of defects and for the CL kink depinning case - on two neighboring horizontal rows of defects. After that we find a sequence of metastable equilibrium meniscus states, simulating plate immersion and withdrawal in the pool of liquid. To this end we move vertically the solid plate in small steps $\pm \Delta z$ ("+" for the study of the receding and "-" of the advancing case). Together with the plate we move also the liquid meniscus, obtained as the equilibrium meniscus state for the previous position of the plate. We use it as an initial state to find through minimization the new equilibrium meniscus for the new plate position. This process is repeated until the CL remains connected with the 
same row(s) of defects, to which it was connected initially. The last found equilibrium meniscus states (corresponding to withdrawal/ immersion of the plate) before a CL depinning occurs, are the ones determining the hysteresis interval.

We note here, that the CAs $\theta_{a d v}$ and $\theta_{r e c}$ in the block-depinning case are obtained analytically [8] when $A / \lambda<<1$ :

$$
\begin{aligned}
& \theta_{a d v}=\pi / 2+\pi^{2}(A / \lambda)^{2}+O\left(A^{3} / \lambda^{3}\right) \\
& \theta_{r e c}=\pi / 2-\pi^{2}(A / \lambda)^{2}+O\left(A^{3} / \lambda^{3}\right)
\end{aligned}
$$

From Eqs. (4) and (5) it follows that

$$
\theta_{a d v}=\pi-\theta_{r e c}
$$

This result holds true without the assumption $A / \lambda<<1$ for the system, shown in Fig. 1, with free energy (2) and local equilibrium CA $\theta_{e q}=90^{\circ}$. This key statement is based on the existence of the "Corresponding states" [9].

\section{Results and discussion}

In Fig. 2 we present $\theta_{\text {rec }}$ (shown by solid symbols and solid line) as function of the ratio $A / \lambda$ and $\pi-\theta_{a d v} \equiv \theta_{r e c}$ (as it follows from Eq. 6).

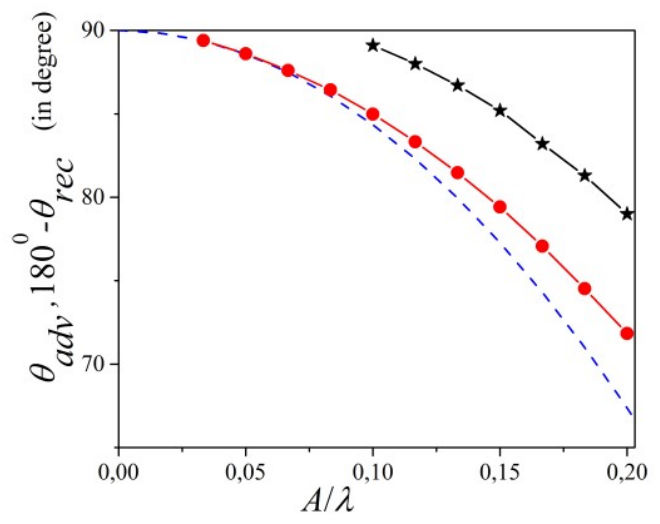

Fig. 2. The ACA and the RCA as functions of the ratio $A / \lambda$. In the CL block-depinning regime the results are shown by circles, connected by solid lines and in the CL kink-depinning regime by stars, connected by solid lines. The analytical results (dashed line) are shown too.

The results for the receding CA $\theta_{\text {rec }}$ are obtained by the procedure, described in Section 2.3. In the CL block depinning regime the results are shown by circles and in the CL kink depinning regime - by stars. The analytical results (Eqs. 4, 5) are shown in Fig. 2 by the dashed line. Our analysis of the presented numerical data shows that all obtained results for 
$\theta_{r e c}$ and $\theta_{a d v}$ as functions of the ratio $A / \lambda$ are approximated well by a quadratic functions. In the CL block-depinning case the numerical results for $\theta_{a d v}$ are smaller and for $\theta_{\text {rec }}$ are bigger than the respective asymptotic solutions. The asymptotic solutions provide good results for $A / \lambda<0.1$. The difference between the numerical solutions and the asymptotic solutions increases with the ratio $A / \lambda$. The difference in the values of $\theta_{a d v}$ (and in the values of $\theta_{\text {rec }}$ ) for both regimes is almost constant and is of the order of $5^{0}-7^{0}$ and slowly increases with the increase of $A / \lambda$. The values of the CAH are respectively by $10^{\circ}-14^{\circ}$ smaller for all considered values of the ratio $A / \lambda$. When the CAH in the CL block-depinning case is smaller than $10^{\circ}$, then in the CL kink-depinning case we do not find $\mathrm{CAH}$.

\section{Conclusion}

Different stick-slip regimes of the CL appear, depending on the mutual disposition of the $\mathrm{CL}$ and the lattice of periodic defects. This leads to different values of the CAH. One needs to investigate both the CL block- and the CL kink-dewetting regimes for a complete study of the CAH in the system, and to take into account also the possible different positions of the defects lattice in respect to the liquid front. When a CL kink-dewetting mechanism is taking place in the system, there is no $\mathrm{CAH}$ at small values of the ratio $A / \lambda$.

\section{References}

1. L. W. Schwartz, S. Garoff, J. Colloid Interface Sci. 106, 422 (1985)

2. M. Rivetti, J. Teisseire, and E. Barthel, Phys. Rev. Lett. 115, 016101 (2015)

3. S. Iliev, and N. Pesheva, Phys. Rev. E 93, 062801 (2016)

4. S. Iliev, Comput. Methods Appl. Mech. Engrg. 126, 251 (1995)

5. S. Iliev, and N. Pesheva, J. Colloid Interface Sci. 301, 677 (2006)

6. D. Iliev, N. Pesheva, and S. Iliev, Langmuir 29, 5781 (2013)

7. K. Brakke, Exp. Math. 1, 141 (1992)

8. R. G. Cox, J. Fluid Mech. 131, 1 (1983)

9. A. D. Myshkis, V. G. Babskii, N. D. Kopachevskii, L. A. Slobozhanin and A. D. Tyuptsov, Low Gravity Fluid Mechanics (Springer-Verlag, Berlin, 1987) 